\title{
Получение электроимпульсным плазменным спеканием термоэлектрических материалов на основе Si и Ge
}

\author{
() И.В. Ерофреева ${ }^{1}$, М.В. Дорохин ${ }^{1}$, А.В. Здоровейщев ${ }^{1}$, Ю.М. Кузнецов ${ }^{1,2}$, \\ A.А. Попов ${ }^{1,2}$, Е.А. Ланцев ${ }^{2}$, А.В. Боряков ${ }^{3}$, В.Е. Котомина ${ }^{1}$ \\ ${ }^{1}$ Научно-исследовательский физико-технический институт \\ Нижегородского государственного университета им. Н.И. Лобачевского, \\ 603950 Нижний Новгород, Россия \\ ${ }^{2}$ Нижегородский государственный университет им. Н.И. Лобачевского, \\ 603950 Нижний Новгород, Россия \\ ${ }^{3}$ Научно-исследовательский институт химии \\ Нижегородского государственного университета им. Н.И. Лобачевского, \\ 603950 Нижний Новгород, Россия \\ E-mail: irfeya@mail.ru
}

(Получена 25 апреля 2018 г. Принята к печати 7 мая 2018 г.)

\begin{abstract}
Исследованы термоэлектрические материалы на основе $\mathrm{SiGe,} \mathrm{полученного} \mathrm{электроимпульсным} \mathrm{плазмен-}$ ным спеканием. Проведено варьирование степени легирования исходных материалов, степени перемешивания $\mathrm{Si}$ и $\mathrm{Ge}$, а также свойств зеренной структуры сформированных образцов. Установлено, что использование исходных материалов, легированных донорными или акцепторными примесями позволяет управлять концентрацией носителей заряда в спеченных образцах. Показано, что концентрация носителей оказывает наиболее существенное влияние на энергетические характеристики термоэлектриков (фактор мощности). Для структур с наибольшей концентрацией носителей управление термоэлектрическими коэффициентами достигается путем варьирования степени перемешивания $\mathrm{Ge}$ и $\mathrm{Si}$, а также однородности распределения примесей.
\end{abstract}

DOI: $10.21883 /$ FTP.2018.12.46756.35

\section{1. Введение}

Полупроводниковый материал на основе $\mathrm{Si}$ и $\mathrm{Ge}$ является широко известным высокотемпературным термоэлектриком [1]. Современные тенденции технологии термоэлектриков связаны с созданием материалов с мелкозернистой и ультрамелкозернистой поликристаллической структурой, которая обеспечивает низкие значения теплопроводности при сохранении высоких значений коэффициента Зеебека и электропроводности $[2,3]$. В отличие от монокристаллов показатели эффективности поликристаллических термоэлектриков зависят от размеров зерна, свойств границ зерен, степени перемешивания $\mathrm{Ge}$ и $\mathrm{Si}$ в твердом растворе. Контроль указанных параметров обеспечит управление свойствами термоэлектрических преобразователей энергии.

В настоящей работе исследованы материалы на основе $\mathrm{Si}$ и $\mathrm{Ge}$, полученные методом электроимпульсного плазменного спекания (ЭИПС) порошков [4]. Ранее в серии работ [5-9] было продемонстрировано, что формирование поликристаллических твердых растворов $\mathrm{Si}_{1-x} \mathrm{Ge}_{x}$ методом ЭИПС позволяет получать термоэлектрические преобразователи энергии с рекордными значениями термоэлектрической добротности. Указанный метод предоставляет широкие возможности для контроля параметров поликристаллической структуры и позволяет эффективно управлять всеми основными характеристиками термоэлектриков. Особенностью применения метода ЭИПС для формирования термоэлектрических преобразователей на основе $\mathrm{Si}_{1-x} \mathrm{Ge}_{x}$ является способ легирования, который заключается в добавлении к порошкам исходных материалов порошков фосфора (для придания электронной проводимости) или примеси бора (для придания дырочной проводимости) с концентрацией $\sim 1-3$ ат\% в обоих случаях [4-9]. Предполагается, что при соответствующем выборе режимов спекания происходит эффективное перемешивание материалов и однородное легирование твердого раствора донорной или акцепторной примесью до уровня, необходимого для получения высокоэффективных преобразователей (как правило, максимальная эффективность характерна для сильно легированных полупроводников [1]).

Отличительной особенностью данной работы является альтернативный способ введения примеси в спекаемые порошки, а именно использование в качестве исходных материалов германия и кремния, сильно легированных донорной примесью. При подборе одинакового типа и уровня легирования $\mathrm{Si}$ и Ge равномерное распределение примеси в этом случае может быть обеспечено уже на этапе формирования порошков для спекания. Важным вопросом при выборе такого способа легирования в методе ЭИПС является оценка структурных свойств, степени перемешивания $\mathrm{Ge}$ и $\mathrm{Si}$, а также процессов диффузии примесей в ходе спекания на электрофизические и термоэлектрические свойства: электропроводность, коэффициент Зеебека, фактор мощности. Для изучения указанного вопроса нами была изготовлена серия образцов $\mathrm{Si}-\mathrm{Ge}$, спеченных при разных условиях, а также с варьированием легирования исходных материалов. 
Параметры механической активации и спекания

\begin{tabular}{c|l|l|c}
\hline № образца & \multicolumn{1}{|c|}{ Условия размола } & \multicolumn{1}{|c}{ Условия спекания } & Характер проводимости \\
\hline 1 & $\mathrm{Si}: \mathrm{As}, \mathrm{Ge}: \mathrm{As}$ & $1020^{\circ} \mathrm{C}$ & Металлический \\
2 & $\mathrm{Si}: \mathrm{As}, \mathrm{Ge}: \mathrm{As}$ & $930^{\circ} \mathrm{C}+$ доп. спекание при $T=1200^{\circ} \mathrm{C}$ с расплавлением & То же \\
3 & $\mathrm{Si}: \mathrm{As}, \mathrm{Ge}: \mathrm{As}$ & $930^{\circ} \mathrm{C}$ доп. спекание при $T=1200^{\circ} \mathrm{C}$ с расплавлением & $\gg$ \\
4 & $\mathrm{Si}: \mathrm{B}$ (КДБ-10), Ge:As & $\begin{array}{l}\text { и отжиг в течение } 10 \text { мин } \\
\text { 970 }\end{array}$ & Полупроводниковый
\end{tabular}

\section{2. Материалы, образцы и их изготовление}

В работе исследованы 4 структуры, полученные при разных условиях спекания. Исходным материалом для структур 1-3 являлся лом монокристаллических подложек $\mathrm{Ge}$ и $\mathrm{Si}$, сильно легированных донорной примесью (As) до концентрации $\sim 10^{19} \mathrm{~cm}^{-3}$. В образце 4 выполнялось искусственное снижение концентрации носителей заряда, поэтому в качестве исходного материала для Si-компоненты использовались подложки, легированные бором (КДБ-10) до концентрации $\sim 2 \cdot 10^{15} \mathrm{~cm}^{-3}$. Ожидалось, что использование акцепторной примеси позволит снизить концентрацию электронов в спеченном материале.

Порошок для спекания изготавливался путем механической активации: размалывания пластин $\mathrm{Ge}$ и $\mathrm{Si}$ c помощью мельницы Fritsch. Полученный порошок помещался в графитовую пресс-форму и подвергался электроимпульсному плазменному спеканию при разных температурах (см. таблицу). Состав спеченного образца соответствовал соотношению $\mathrm{Si}_{0.8} \mathrm{Ge}_{0.2}$, которое задавалось путем взвешивания исходных материалов и последующего пересчета из весовых процентов в атомные.

Использовано два режима спекания. Образцы 1 и 4 спекались в один этап при одной температуре $\left(1020^{\circ} \mathrm{C}\right.$ для образца 1 и $970^{\circ} \mathrm{C}$ для образца 4). Образцы 2 и 3 спе-

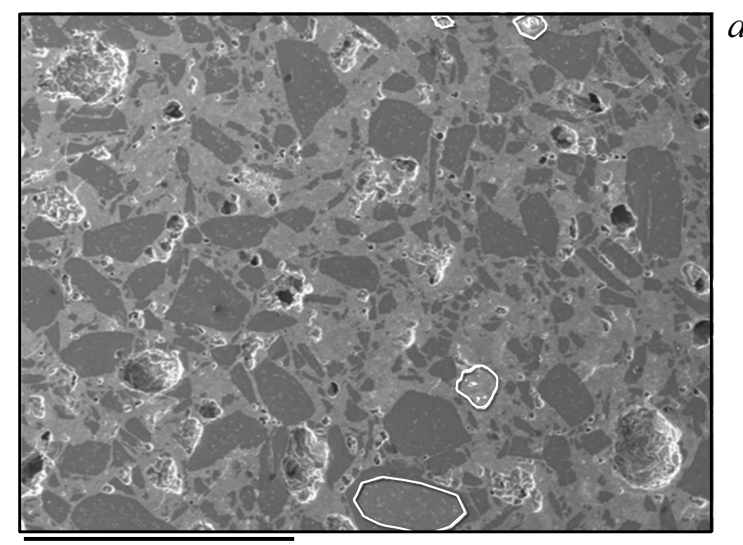

$1 \mathrm{~mm}$

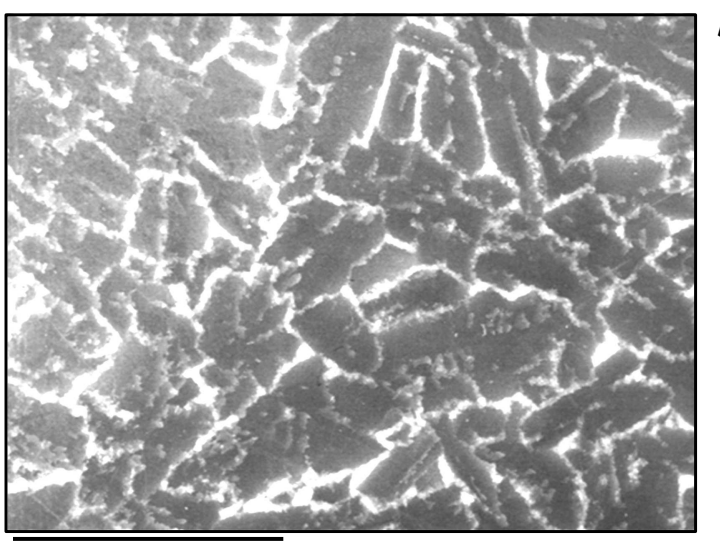

$1 \mathrm{~mm}$

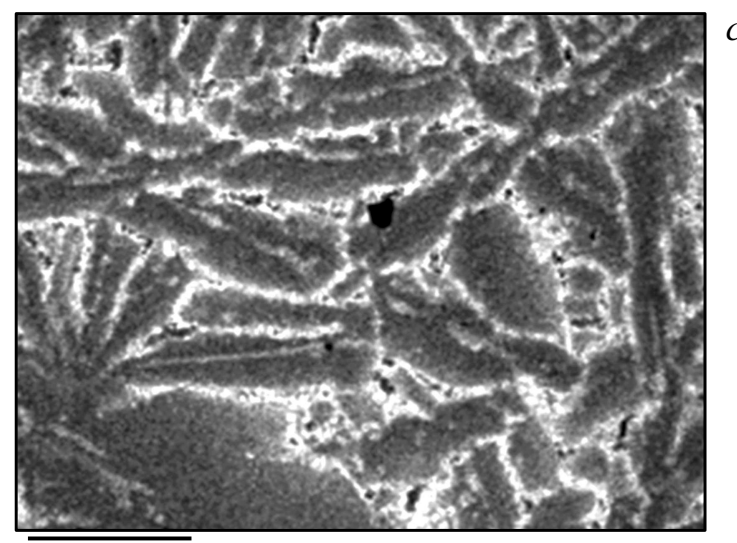

$500 \mu \mathrm{m}$

Рис. 1. Электронно-микроскопическое изображение поверхности: $a-$ образец $1, b-$ образец $2, c-$ образец 3 . Белыми линиями выделены области, в которых выполнено измерение состава для определения степени перемешивания. 
кались в два этапа: на первом этапе выполнялась предварительная усадка порошка при $T=930^{\circ} \mathrm{C}$. На втором этапе образцы помещались в новую (большего размера) форму и дополнительно спекались при более высокой температуре $T=1200^{\circ} \mathrm{C}$, предположительно, с расплавлением. Образец 3, кроме того, дополнительно выдерживался при высокой температуре в течение 10 мин. Интегральный состав полученного материала определялся методом рентгеновского микроанализа с использованием сканирующего электронного микроскопа Jeol JSM-IT300LV и энергодисперсионной приставки Oxford Instruments $\mathrm{X}-\mathrm{Max}^{\mathrm{N}} 20$ и соответствовал рассчитанному и приготовленному весовому соотношению $\mathrm{Si}$ и $\mathrm{Ge}$ материала $\left(\mathrm{Si}_{0.8} \mathrm{Ge}_{0.2}\right)$.

Спеченные объемные образцы были разрезаны на слитки с размерами (длина/ширина/высота) $10 \times 5 \times 3$ мм. Одна из поверхностей слитка подвергалась механической шлифовке и полировке. Для измерения термоэлектрических характеристик на поверхность образцов наносились металлические омические контакты.

Перед нанесением контактов шлифованные поверхности образцов были исследованы с применением сканирующего электронного микроскопа Jeol JSM-IT300LV (оборудование ЦКП „Новые материалы и ресурсосберегающие технологии“ ННГУ им. Н.И. Лобачевского).

На электронно-микроскопическом изображении поверхности образца 1 (рис. $1, a$ ) видны области различающегося контраста, характерные размеры которых составляют 150-600 мкм. Измерение состава методом рентгеновского микроанализа (не приведено) показало, что указанные области соответствуют несмешанным $\mathrm{Ge}$ и Si. Таким образом, образец 1 представляет собой спрессованную структуру, состоящую из сравнительно крупных зерен Ge и Si.

Для образцов 2 и 3 зарегистрировано некоторое укрупнение зерен до $\sim 200-500$ мкм. Различие между контрастом отдельных зерен практически не наблюдается. Отличаются контрастом лишь области границ. Измерение состава методом рентгеновского микроанализа показало наличие в зернах как атомов $\mathrm{Ge}$, так и $\mathrm{Si}$, следовательно, в режимах спекания, выбранных для образцов 2 и 3, произошло перемешивание материалов и формирование зеренной структуры сплава $\mathrm{GeSi}$.

\section{3. Измерение термоэлектрических характеристик}

Были исследованы температурные зависимости удельного сопротивления $(\rho)$ и термоэдс. Для измерения низких значений удельного сопротивления структур использовалась четырехзондовая методика. Величина термоэдс $\left(U_{\mathrm{TP}}\right)$ различалась для разных структур, но для всех исследованных образцов знак $U_{\mathrm{TP}}$ соответствует проводимости $n$-типа (потенциал „горячего“ конца образца выше потенциала „холодного“ [3]). По полученным данным был рассчитан коэффициент Зеебека $\alpha$

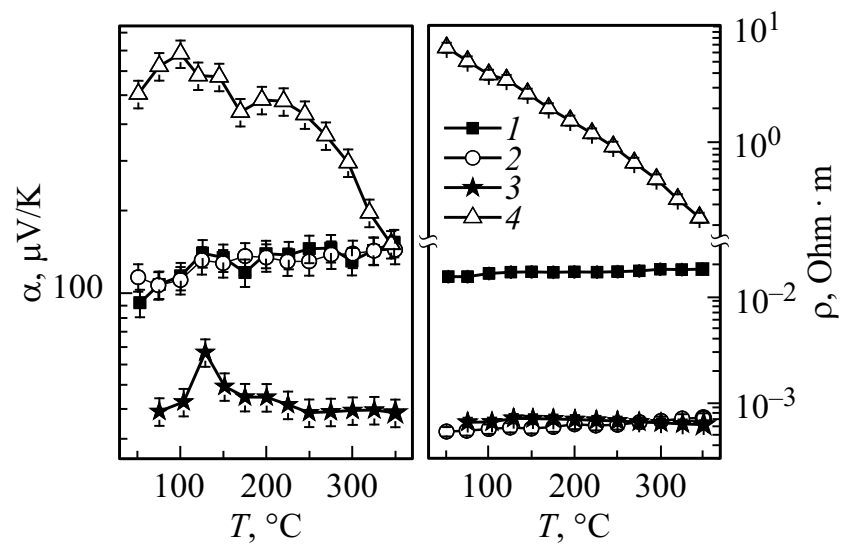

Рис. 2. Зависимость коэффициента Зеебека $(a)$ и удельного сопротивления $(b)$ от температуры $T$ (номер линии соответствует номеру образца).

для различных образцов в зависимости от температуры $\alpha=U_{\mathrm{TP}} / \Delta T$. Температурные зависимости коэффициента Зеебека и удельного сопротивления образцов представлены на рис. 2.

Наибольшие значения термоэдс были получены для образца 4, сформированного с компенсацией донорного легирования. Для указанной структуры также были получены наибольшие значения удельного сопротивления, сама величина сопротивления существенно снижается с ростом температуры (полупроводниковый характер проводимости).

Для структур 1-3, сформированных из сильно легированных донорной примесью материалов, температурные зависимости удельного сопротивления и коэффициента Зеебека подобны, различие заключается лишь в величинах указанных параметров, что будет обсуждаться далее. Величина сопротивления для структур 1-3 незначительно увеличивается с ростом температуры, что соответствует металлическому характеру электропроводности и связывается с сильным легированием исходных материалов.

Наиболее характерные различия между термоэлектрическими свойствами образцов получены при расчете значения фактора мощности. Фактор мощности - это одна из основных характеристик термоэлектриков, которая рассчитывается по формуле $\alpha^{2} / \rho$. Температурные зависимости фактора мощности для всех исследованных структур приведены на рис. 3. В целом значение фактора мощности слабо зависит от температуры, с ростом $T$ имеет место незначительный рост. Можно отметить существенные, на порядок величины, различия между значениями фактора мощности для исследованных структур. Наибольшие значения получены для образца 2 $\left(\sim 2 \cdot 10^{-4} \mathrm{BT} / \mathrm{M} \cdot \mathrm{K}^{2}\right)$, сформированного с расплавлением порошка. Дополнительный отжиг GeSi-материала (образец 3) приводит к снижению на порядок величины фактора мощности по сравнению с образцом 2. Еще 


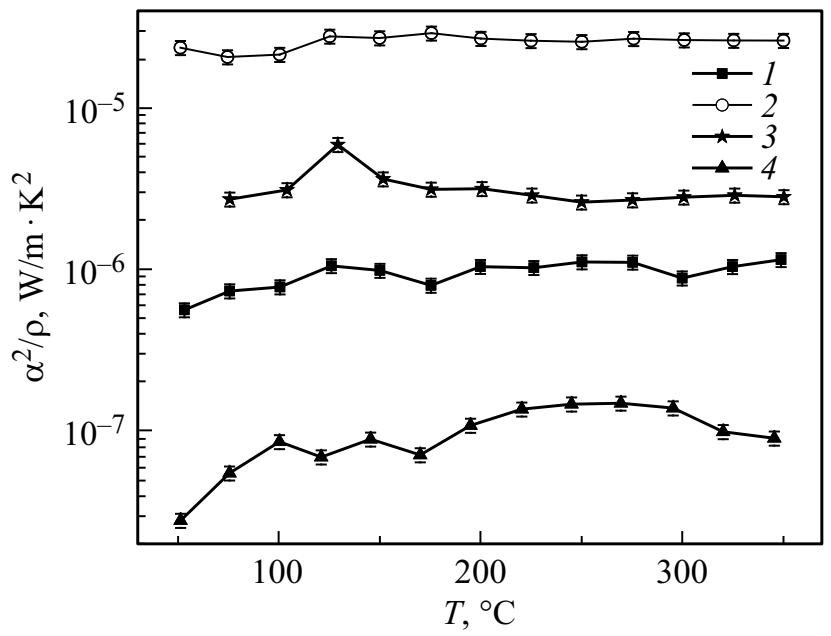

Рис. 3. Зависимость рассчитанного значения фактора мощности от температуры $T$ (номер линии соответствует номеру образца).

более низкое значение фактора мощности было получено для образца 1, для которого было установлено отсутствие перемешивания $\mathrm{Ge}$ и $\mathrm{Si}$. Наименьшее значение получено для образца 4 с частичной компенсацией донорной примеси.

\section{4. Обсуждение результатов}

Полученные различия термоэлектрических свойств мы связываем со структурой сформированных образцов, а также с особенностями их легирования. Образец 1, как было показано выше (рис. 1,a), представляет собой спрессованную структуру, состоящую из сравнительно крупных несмешанных зерен $\mathrm{Ge}$ и $\mathrm{Si}$. В такой структуре резистивные характеристики определяются широкозонным материалом ( $\mathrm{Si}$, который имеет более высокое удельное сопротивление), а коэффициент Зеебека - узкозонным (Ge). Отсутствие смешивания обусловливает наибольшие значения электросопротивления, при этом принципиального повышения коэффициента Зеебека не происходит.

В образце 2 смешивание материалов, по-видимому, имело место. В результате термоэлектрические свойства образца 2 соответствуют свойствам твердого раствора $\mathrm{Si}_{0.8} \mathrm{Ge}_{0.2}$. Для указанной структуры управление сопротивлением и коэффициентом Зеебека может быть достигнуто путем изменения размера зерна (для выбранного режима спекания размер зерна сравнительно крупный).

Интересным экспериментальным фактом является снижение коэффициента Зеебека для структуры 3, подвергнутой дополнительному высокотемпературному отжигу в процессе спекания. При этом существенной модификации зеренной структуры не произошло, как свидетельствуют микрофотографии (рис. $1, b$ и $c$ ), также не произошло значительного изменения электросопротивления (рис. 2, $b$, кривые 2 и 3 ).

Можно предположить частичную сегрегацию примеси As на границы зерен: для подтверждения этой гипотезы были выполнены измерения карт распределения мышьяка по поверхности. Измерения выполнены методом рентгеновского микроанализа, результаты представлены на рис. 4. Для образцов 1 и 2 зарегистрировано однородное латеральное распределение атомов мышьяка. Локальные максимумы (яркие точки на карте распределения элементов) обусловлены большой относительной погрешностью метода при определении концентраций вблизи 1 ат\%. Для образца 3 зарегистрированы области повышенной концентрации атомов мышьяка, причем положение этих областей хорошо коррелирует с положением границ зерен (ср. рис. 1, $c$ и рис. 4, b).

Таким образом, карты распределения элементов, на наш взгляд, являются подтверждением тезиса о скапливании атомов легирующей примеси на границах зерен. Предположительно, влияние данного эффекта на величину сопротивления невелико (в силу повышения роли электропереноса по низкоомным границам зерен), при этом в силу снижения концентрации примеси в самих $\mathrm{SiGe-зернах} \mathrm{величина} \mathrm{термоэдс} \mathrm{снижается.} \mathrm{Это}$ объясняет снижение фактора мощности для образца 3 по сравнению с образцом 2.

Наконец, наиболее существенное снижение фактора мощности зарегистрировано для образца 4, в котором осуществлялась частичная компенсация примеси As примесью бора. Это привело к существенному снижению концентрации носителей, что в свою очередь обусловливает снижение фактора мощности [10]. Параметры зеренной структуры оказывают меньшее влияние на термоэлектрические коэффициенты и их изменение позволяет повышать термоэлектрические коэффициенты лишь в случае максимального легирования структур.

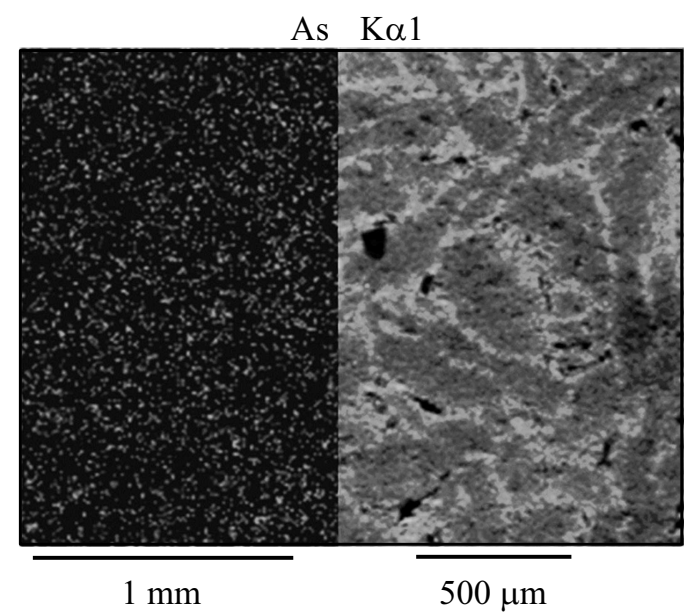

Рис. 4. Результаты рентгеновского микроанализа: $a$ - образец $1, b$ - образец 3. 


\section{5. Заключение}

Таким образом, в работе исследованы термоэлектрические материалы на основе $\mathrm{SiGe}$, полученного электроимпульсным плазменным спеканием. Проведено варьирование степени легирования материалов, степени перемешивания $\mathrm{Si}$ и $\mathrm{Ge}$, а также свойств зеренной структуры сформированных материалов. Получено, что использование исходных материалов, легированных донорными или акцепторными примесями, позволяет управлять концентрацией носителей заряда в спеченных образцах. Показано, что концентрация носителей оказывает наиболее существенное влияние на энергетические характеристики термоэлектриков (фактор мощности). Для структур с наибольшей концентрацией носителей показана возможность управления величиной фактора мощности за счет варьирования зеренной структуры и пространственного распределения примеси в сформированной поликристаллической структуре.

Работа выполнена при поддержке Российского научного фонда (проект № 17-79-20173).

\section{Список литературы}

[1] Termoelectric hand book macro to nano, ed. by D.M. Rowe (CRC Press, Boca Raton, 2006).

[2] L. Hicks, M. Dresselhaus. Phys. Rev. B, 47, 16631 (1993).

[3] C. Gayner, K.K. Kar. Prog. Mater. Sci., 47, 330 (2016).

[4] B. Yu, M. Zebarjadi, H. Wang, K. Lukas, H. Wang, D. Wang, C. Opeil, M. Dresselhaus, G. Chen, Z. Ren. Nano Lett., 12, 2077 (2012).

[5] S. Bathula, M. Jayasimhadri, A. Dhar. Mater. Des., 87, 414 (2015).

[6] R. Murugasami, P. Vivekanandhan, S. Kumarana, R. Suresh Kumar, T. John Tharakan. Scripta Mater., 143, 35 (2018).

[7] D. Thompson, D. Hitchcock, A. Lahwal, T.M. Tritt. Emerging Mater. Res., 1, (EMR6), 299 (2012).

[8] Z. Zhu, S. Guo. Key Eng. Mater., 703, 70 (2016).

[9] S. Bathula, M. Jayasimhadri, N. Singh, A.K. Srivastava, J. Pulikkotil, A. Dhar, R.C. Budhani. Appl. Phys. Lett., 101 (21), 213902, (2012).

[10] J.R. Szczech, J.M. Higgins, S. Jin. J. Mater. Chem., 21, 4037 (2011).

Редактор А.Н. Смирнов

\section{The spark-plasma sintering fabrication of $\mathrm{Si}$ and Ge-based thermoelectric materials}

\author{
I.V. Erofeeva ${ }^{1}$, M.V. Dorokhin ${ }^{1}$, A.V. Zdoroveyshchev' ${ }^{1}$, \\ Yu.M. Kuznetsov 1,2, A.A. Popov ${ }^{1,2}$, E.A. Lantsev' ${ }^{2}$, \\ A.V. Boryakov ${ }^{3}$, V.E. Kotomina ${ }^{1}$ \\ ${ }^{1}$ Research Institute for Physics and Technology, \\ Nizhny Novgorod State University, \\ 603950 Nizhny Novgorod, Russia \\ 2 Nizhny Novgorod State University, \\ 603950 Nizhny Novgorod, Russia \\ ${ }^{3}$ Research Institute for Chemistry, \\ Nizhny Novgorod State University, \\ 603950 Nizhny Novgorod, Russia
}

\begin{abstract}
Thermoelectric materials based on SiGe obtained by spark-plasma sintering were investigated. The doping degree of the initial materials, the degree of mixing of $\mathrm{Si}$ and $\mathrm{Ge}$, as well as the properties of the grain structure of the fabricated samples were varied. It was found that the use of initial materials doped with donor or acceptor impurities allows controlling the concentration of carriers in sintered samples. It was shown that the carrier concentration has the most significant influence on the energy characteristics of thermoelectrics (power factor). For structures with the highest carrier concentration, the control of the thermoelectric coefficients was achieved by varying the degree of mixing of $\mathrm{Ge}$ and $\mathrm{Si}$, as well as the uniformity of impurity distribution.
\end{abstract}

